\title{
Evolution and impingement of an automotive fuel spray investigated with simultaneous Mie/LIF techniques
}

\author{
B.D. Stojkovic, V. Sick* \\ Department of Mechanical Engineering, The University of Michigan, 2023 W.E. Lay Automotive Laboratory, 1231 Beal Avenue, Ann Arbor, MI \\ 48109-2121, USA
}

Received: 5 December 2000/Revised version: 28 February 2001/Published online: 23 May 2001 - ( S Springer-Verlag 2001

\begin{abstract}
The spatial and temporal evolution of an automotive hollow-cone-type spray was investigated with laserbased imaging diagnostics. Optical conditions of an IC engine were emulated with a test cell that was built from an engine cylinder head to hold a high-pressure gasoline-fuel injector. The use of iso-octane fuel that was doped with 3-pentanone allowed measurements of laser-induced fluorescence (LIF) after excitation with a $\mathrm{KrF}$ excimer-laser beam. A versatile optical filter system was designed and built that permits simultaneous measurements of Mie-scattering and laser-inducedfluorescence images using a single laser-light sheet and a single intensified CCD camera. The influence of background signals, caused by reflection of signal light from surfaces, laser-sheet intensity attenuation and signal decrease by scattering, was characterized. Mass distributions showed a distinct pre-spray phase, more so than the Sauter mean diameter $(S M D)$ that was determined from the ratio of LIF to Mie signals using single pulse as well as averaged image pairs. Significant changes in $S M D$ distributions were found after the spray had impinged on a flat surface. The impingement also led to the buildup of a liquid film whose thickness was quantitatively determined from LIF images.
\end{abstract}

PACS: $32.50+d ; 39.30+w$

The development of improved spark-ignition gasoline engines is making increased use of direct fuel injection into the cylinder. Due to the limited time that is available for atomization and evaporation of the fuel in an engine cycle before the spark ignites the fuel/air mixture, the spray formation plays a crucial role in the functionality of such engines. If the fuel is not well evaporated and mixed with the air, the combustion process will be affected and low power output as well as increased pollutant levels will be the result. It is therefore necessary to have experimental techniques available that allow a detailed study of spray structures with high temporal and

${ }^{*}$ Corresponding author.

(Fax:+1-734/764-4256, E-mail: vsick@umich.edu) spatial resolution. Parameters of interest are the spatial distribution of droplets, fuel mass and droplet diameters. In cases where the spray hits the piston surface, the thickness of the liquid fuel film and the motion of the spray along the piston surface must be characterized. It is desirable to achieve a simultaneous acquisition of two or more physical parameters at any instance in time and space, to allow a more comprehensive description of the instantaneous spray condition. For example, simultaneous planar laser-induced fluorescence (LIF) and Mie-scattering measurements were used to characterize the spray formed by a blast film atomizer and provided information about the evaporation characteristics [1]. While these measurements yielded a qualitative description of the evaporation process, no information was obtained about the droplet diameters. The most common drop-sizing technique for spray characterization is phase Doppler anemometry (PDA) [2]. PDA measurements provide absolute droplet diameters and work well as long as the spray density is not too high [3]. The characterization of an entire spray can take hours due to the scanning nature of a PDA setup, which only measures at one point at a time. Recent studies [4-6] have shown that laser-sheet illumination of a complete spray structure yields the relative Sauter mean diameter $(S M D)$ distribution via the ratio of LIF and Mie-scattering images. Using the ratio of the two signals that are produced by the same laser beam eliminates sources of error, such as laser sheet intensity variations or shot-to-shot variations of the total laser energy, as these would affect both signals in the same way. Yeh et al. [4] first described such an approach, using an optical arrangement with a roof prism and filters to split LIF from Mie signals. The spatially separated signals were then imaged onto a single CCD camera. Zelina et al. [5] recorded averaged images of Mie scattering and LIF in sequence, using a single camera. Thus, no information on instantaneous spray structures can be obtained. Rachner et al. [1] used reflective optics to spectrally and spatially separate Mie-scattering and LIF signals and used two intensified CCD (ICCD) cameras for detection. While this can provide instantaneous images, no effort was made to calculate the ratio of LIF to Mie signals and thereby gaining information about the droplet diameters. Jermy and Greenhalgh [6] used sequential detection of Mie 
and LIF of a water spray that was doped with sodium salicylate as fluorescent dye and compared the results of the $S M D$ determination with PDA measurements. They systematically characterized sources and magnitudes of uncertainty for the ratio-based droplet-diameter measurement and concluded that the method has improved capabilities compared to PDA albeit the inherent need for external calibration, e.g. with a PDA measurement.

A flexible filter setup for spatial separation of spectrally distinguishable signals is described in this study for the use of simultaneous recording of e.g. Mie and LIF signals for $S M D$ determination. The advantages of this filter are a simple and robust setup, unambiguous signal isolation, independent focus adjustment for the separated signals and simple modifications for other optical applications. The description of the design and calibration of the filter optics, as well as the laser-sheet arrangement with counter-propagating beams, is followed by a discussion of disturbances that could influence spray measurements in a realistic engine-like environment. The applicability of the filter setup is demonstrated with measurements of relative Sauter mean droplet diameters of a hollow-cone automotive fuel spray. These measurements were performed in a freely propagating spray as well as a spray that impinged on a flat surface. Other quantities that were determined are the thickness of the liquid-fuel film that forms upon impingement of the spray on the flat plate and the extent of the expanding spray plume.

\section{Spray setup and dual-signal acquisition optics layout}

The experiments were conducted in an optical test cell, which mimics optical features of a transparent research engine. A high-pressure hollow-cone fuel injector (Zexel) that was mounted into a single-cylinder engine cylinder head was used to produce the sprays. The nominal spray cone angle of this injector was quoted by the manufacturer as $30^{\circ}$. The fuel was pressurized to $6.5 \mathrm{MPa}$ with a piston accumulator and then injected into the test cell that was held at atmospheric pressure and room temperature. During the injection duration of $1.2 \mathrm{~ms}$ (electric pulse duration), $14 \mathrm{mg}$ of fuel were injected at a frequency of $1 \mathrm{~s}^{-1}$. Injection timing, laser triggering and ICCD camera control were controlled with a digital pulse/delay generator (SRS DG535). The times that are reported subsequently in this paper refer to time after the trigger pulse was sent to the injector driver. This means that the first appearance of fuel is somewhat later due to delays within the injector driver and mechanical properties of the injector. These times typically are on the order of a few tenths of a millisecond. Fuel pressure and injection duration remained the same for all experiments discussed here. Isooctane was used as fuel in the experiments. For fluorescence visualization, the iso-octane was doped with $0.5 \%$ (vol.) 3pentanone as a fluorescent tracer. 3-pentanone was chosen because of its well-defined fluorescence behavior [7,8]. The LIF spectrum of 3-pentanone is broadband, from $330 \mathrm{~nm}$ to $600 \mathrm{~nm}$, with a peak at $430 \mathrm{~nm}$. The absorption spectrum of 3 -pentanone ranges from $240-320 \mathrm{~nm}$ and is characterized with a peak at $280 \mathrm{~nm}$. Absorption and emission are spectrally well separated, ensuring the absence of fluorescence trapping and offering the possibility of separating Mie scattering and fluorescence. A KrF excimer laser (Lambda Physik EMG
150 TMSC), producing 248-nm light, was used for excitation. The laser was operated in single-pass amplification by replacing the standard beam-expanding prisms with taller ones and removing the Cassegrain optics of the amplifier. This produces a smoother intensity profile across the beam. The excimer laser delivers pulses of up to $120 \mathrm{~mJ}$, with approximately 17-ns pulse length and a rectangular cross-section of the beam of 29-mm height and 9.5-mm width at the exit of the laser. Shot-to-shot energy fluctuations of up to $\pm 16 \%$ of the mean pulse energy were measured with a calibrated photodiode (LaVision). The laser beam was split into two beams of roughly equal intensity (1:1.1). The two beams, which are compressed into 0.6 -mm-thick light sheets with cylindrical lenses, entered the engine model cell in a counter-propagating arrangement. Illumination of dense fuel sprays with counterpropagating light sheets has been shown as a suitable way to reduce the effect of strong laser-intensity attenuation that is caused by scattering and/or absorption within the spray [9]. The overall experimental layout is shown in Fig. 1.

The laser-light sheets could be aligned to either illuminate a vertical or horizontal cross-section of the spray. When a horizontal plane was illuminated, the emerging signals were directed to the filter and camera system via a UVenhanced $\mathrm{Al}$ mirror. In the case of a vertical illumination the filter/camera system was moved to the side of the engine cell. The Mie-scattering and LIF components of the signal were spatially separated using a combination of dielectric mirrors and Schott glass filters as shown in Fig. 2.

The combination of three dielectric mirrors, HR-coated for $248 \mathrm{~nm} / 45^{\circ}$ (Laser Optik, Garbsen), efficiently isolates the Mie scattering from the LIF signal. The reflection characteristic of a single mirror is cubed when using a combination of three mirrors, which produces a narrow-width band pass in reflection. LIF signals are transmitted through the first of the HR 248-nm mirrors. Any remaining Mie-scattering signal can then be removed by using a Schott glass filter (WG 385) with the cut-off wavelength at $385 \mathrm{~nm}$. At this point the two signal contributions are separated in space. The spatial positions of the individual optical components were arranged such that the optical path for the LIF and the Mie signals were identical to guarantee focused imaging of both signals. The two components of the detected signal can have very different in-

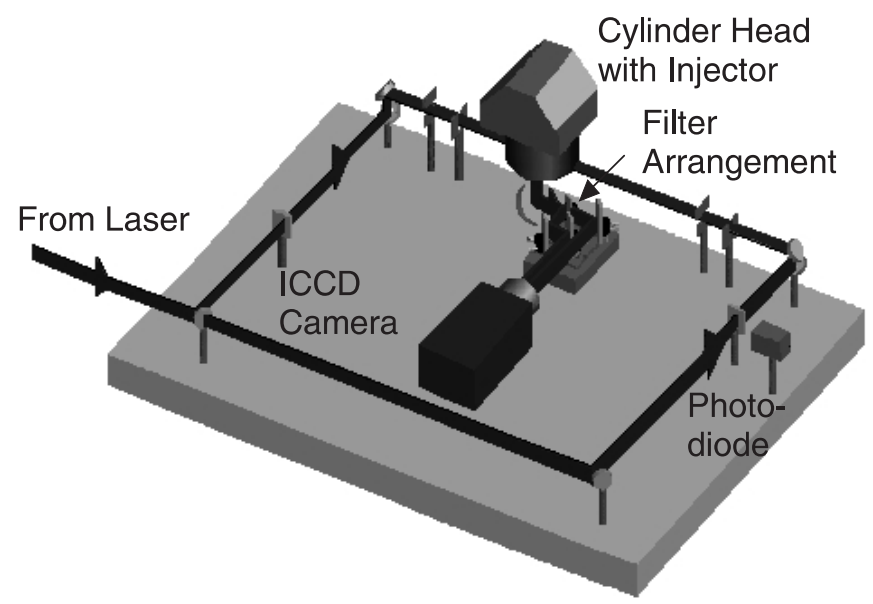

Fig. 1. Measurement of spray features with counter-propagating horizontal laser-light-sheet illumination. The test cell, spray and optical arrangement mimics setups used for measurements in transparent engines 


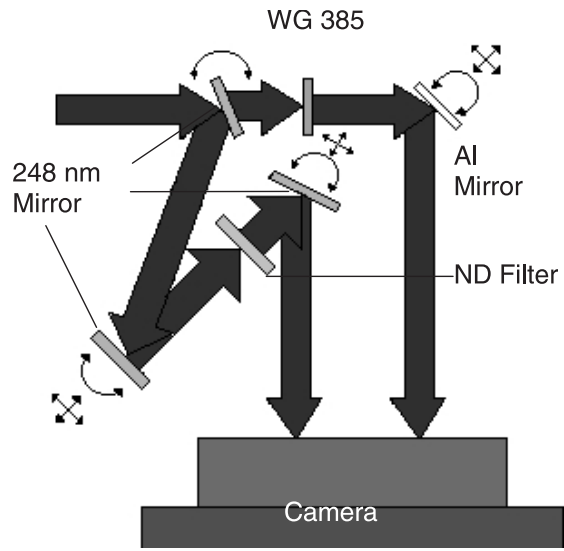

Fig. 2. Imaging filter setup. Standard high-reflectivity dielectric mirrors, coated for maximum reflectivity at $248 \mathrm{~nm}$ under $45^{\circ}$ incidence, isolate Mie-scattering signals. Transmitted LIF signals are filtered with Schott color glass, WG 385 , before being directed to the collecting lens via a UVenhanced Al mirror. The Mie signals can be attenuated with neutral density filters to match the intensity of the LIF signals. The HR mirrors can be translated and rotated to adjust the optical path for the Mie-scattering signals to match the path for the LIF signals

tensity levels. However, the open design of the filter allows the introduction of neutral density filters to match the intensities of Mie and LIF signals. The signals were then imaged onto a 14-bit ICCD camera with $384 \times 576$ pixels (LaVision Flame Star II) using an achromatic UV lens (Halle, $\mathrm{f}_{\#}=2$ ).

A transmission spectrum of the filter system was measured to prove that Mie and LIF signals are fully separated. The filter setup, shown in Fig. 2, was illuminated with collimated light from a broadband high-pressure 75-W Xe lamp (Newport Model 870). The transmitted light was focused onto an imaging spectrograph (Acton Research Corporation SpectraPro-150) with an achromatic UV lens. Signals were then measured with the intensified CCD camera. The wavelength reading of the spectrograph was calibrated using a mercury lamp (Oriel). A baseline for the spectral intensity distribution of the Xe lamp was recorded before inserting the filter system.

The transmission spectrum in Fig. 3 shows that the desired response of the system was achieved. The peak transmission at $255 \mathrm{~nm}$ with a FWHM of $25 \mathrm{~nm}$ matches the requirements for transmitting the Mie signals. The peak wavelength deviates from the center wavelength of the 248-nm HR mirrors

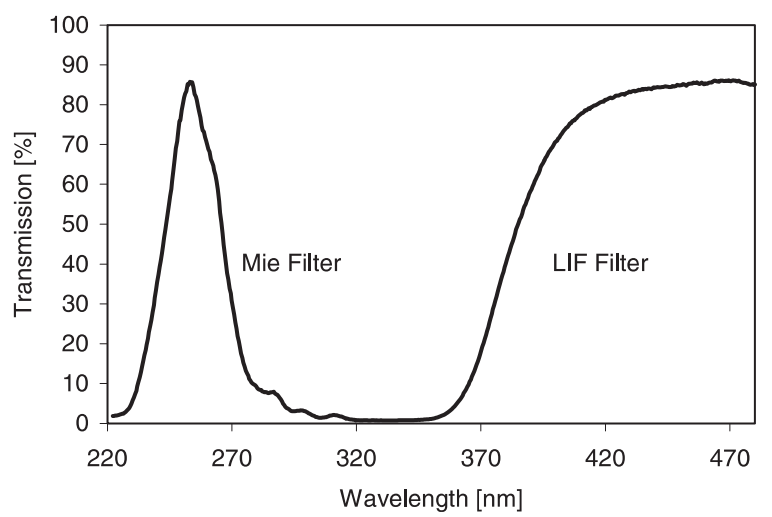

Fig. 3. Measured transmission curve for the filter combination shown in Fig. 2 as these were used at angles somewhat different from $45^{\circ}$ to adjust the optical path length for the Mie signals to the path length for the LIF signals. Although this leads to a reduced transmission at $248 \mathrm{~nm}$, this is not a disadvantage, as the Mie signals have to be attenuated anyway to match the intensity of the LIF signals. Note that the high transmission of the complete filter arrangement is achieved via the high reflectance of the dielectric mirrors.

With this type of filter and detection setup, simultaneous single-shot measurements of LIF and Mie signals for individual spray events are possible with a single laser and a single camera. It is worth mentioning that by simply replacing the three dielectric mirrors and/or the color glass filter, other filtering characteristics can easily be obtained. In the subsequent sections of this paper, a variety of measurements are presented where the described filter has been used.

\section{Detrimental influences on spray-imaging measurements}

Imaging-based measurements of spray structures can suffer from a range of problems, which are addressed below. With the laser sheet illuminating a horizontal cross-section of the spray, $20 \mathrm{~mm}$ below the injector nozzle, pairs of Mie/LIF images were taken. These images show significant scatteredlight intensity that visualizes valves and other features of the cylinder head, which are located substantially above the light-sheet plane. It was determined that the signal light was reflected by the surface of the cylinder head, partially masking the detection of the desired signal. The interference was particularly strong for the LIF signals. It was found that the surface of the air-exposed aluminum cylinder head reflects more strongly in the visible compared to the ultraviolet spectral range [10]. This is apparent from Fig. 4 where the measurement of the Mie signal is less affected by this problem than the LIF signal. Further arguments for the conclusion that the interference is caused by scattered signal light rather than by scattered laser light that hits the surface and possibly excites fuel traces on the surface is the presence of signals that show valve contours when the fuel is allowed to fully evaporate before the measurement. In such a case there are no droplets from which laser light could be reflected onto the surface. Furthermore, even after carefully cleaning and drying the cylinder head surface the background interference is present during the first injection, thus excluding the possibility of residual fuel on the surface that is excited. Tests with LIF from fully vaporized acetone also show substantial signal reflections from the surface. The use of the $\mathrm{f}_{\#}=2$ lens without any f-stop helps to suppress the detection of the surface-scattered signals by minimizing the depth of field. Vapor blasting the surface produced a fine-grain surface roughness, which diffuses the reflected signal light, to help minimize the background signal. The vapor blasting did not fully reach recessed regions of the cylinder head. These locations, e.g. the valve-seat areas, still produce significant background interference.

The acquired signals were corrected for laser-sheet inhomogeneities, and shot-to-shot variation of laser energy, obtained with a photodiode (LaVision). The laser intensity was kept at around $5 \mathrm{MW} / \mathrm{cm}^{2}$, in order to ensure linear LIF conditions [8, 11]. 


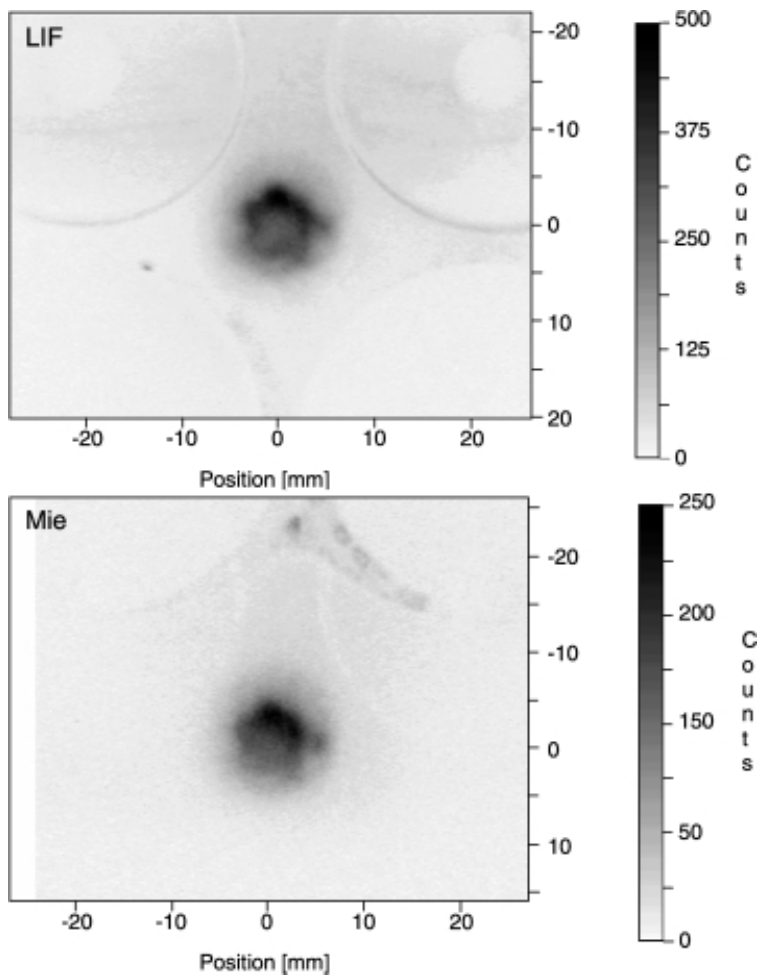

Fig. 4. Signal reflections from the cylinder-head surface significantly distort the LIF signal while a lesser effect on the Mie signal is noticed. The images show averages of 10 individual exposures from a horizontal cross-section $0.86 \mathrm{~ms}$ after start of injection control (ASOIC), $20 \mathrm{~mm}$ below the injector nozzle

The most significant factors that could cause additional inaccuracies in spray measurements are extinction of the laser sheet by scattering and/or absorption as the beam traverses through the spray and attenuation of the signal light between the laser sheet and the camera by the same effects. This could cause significant distortion in the acquired signals, and the measured intensity distribution would not represent the true fuel distribution. Different methods were discussed in the literature to overcome the problem of laser-light attenuation. The simplest approach, shown by Preussner et al. [9], used bidirectional excitation with counter-propagating laser sheets. This led to spray images with a more accurate representation of the true structure. Sick and Stojkovic [12] systematically assessed the validity and limitations of this method. Alternatively, the measured overall attenuation of the laser sheet can be used to reconstruct the true signal intensity with a computational procedure and a stepwise reconstruction of the original laser intensity as described by Su et al. [13]. Talley et al. [14] used sequential illumination of a spray from opposing directions and then applied an iterative reconstruction algorithm to recover the true spray structure.

The following discussion shows the magnitude of attenuation effects on excitation and detection for the present hollowcone spray. The LIF signal is chosen here for the discussion of the laser-light extinction effects. Averaged images $(N=10)$ as shown in Fig. 4 were used to evaluate the influence of laserbeam attenuation on imaging of the particular spray under study. With a counter-propagating beam arrangement both laser beams contribute to the measured signal in an additive way and reduce the distorting effect of laser-beam attenua- tion. The profiles in Fig. 5 show that the signal intensities measured with sequential uni-directional, but opposite, illumination do suffer from extinction effects. Note that the sum of these signals is in close agreement with the result from bi-directional illumination despite the small number (10) of images that were used to calculate the average. It appears that signal losses due to the attenuation on either side of the spray are recovered to a large extent with bi-directional illumination. Both spray 'shoulders' seem to have been recovered in comparison to the results obtained with unidirectional illumination. It is also evident that fuel is found inside of the hollow cone.

In addition to the attenuation of the laser beam as it traverses through the spray, the measured signal is affected by extinction of the signal between the illuminated portion of the spray and the camera. The droplets between the illuminated region and the camera could absorb or scatter off the signal light, causing secondary fluorescence and reflection of light out of the detection optics path, thereby diminishing the actual measurement signal. Since the absorption spectrum of 3-pentanone does not overlap with its fluorescence spectrum, fluorescence trapping is avoided. However, scattering from the droplets can attenuate the LIF signals. The level of light extinction was investigated for a vertical cross-section of the spray. Approximately the first $20-\mathrm{mm}$ downstream of the spray could not be imaged because the pent roof shape of the cylinder head with the injector mounted in the center prevents optical access to the nozzle tip from the side.

The experiment used a dye-laser cuvette placed behind the spray, containing $0.5 \%$ 3-pentanone doped to iso-octane, as illustrated in Fig. 6. A vertical laser-light sheet illuminated the cuvette and generated LIF of 3-pentanone. These LIF signals were attenuated on their path through the spray and were then recorded with an ICCD camera. Using this approach, the transmission measurement is performed for light of the same spectral contents as the LIF signal from the spray.

The 2D transmission image, shown in Fig. 7a, was obtained from an average of 15 individual images by illuminating the cuvette behind the spray at fixed times, $0.86 \mathrm{~ms}$, after the start of injection control and normalizing this image

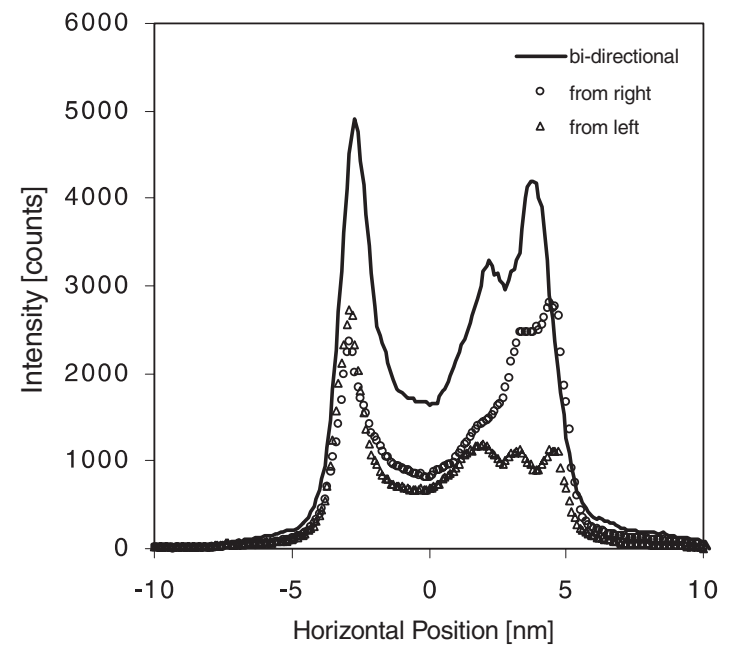

Fig. 5. Cross-sections of averaged LIF profiles for different illumination setups. Note that the profiles are taken from averaged LIF images $(N=10)$ of different spray events. Conditions match the description shown in Fig. 4 


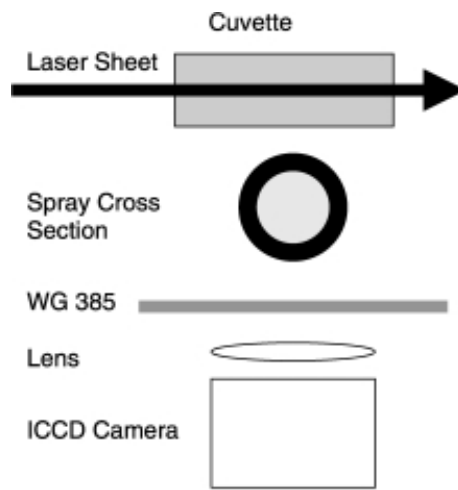

Fig. 6. Schematic top view of the setup that was used to quantify the LIFsignal attenuation through a hollow-cone spray


Fig. 7a,b. Effect of the spray on measured LIF-signal intensities for a vertical cross-section of a hollow-conespray. a Measured LIF and 2Dtransmission image for a spray at $0.86 \mathrm{~ms}$ ASOIC. b Horizontal crosssection at $25 \mathrm{~mm}$ below the injector nozzle showing the extent of signal attenuation. Note that for typical laser-sheet illumination in the center of the spray, the LIF signals will be attenuated by half of the value shown

to an averaged image of the fluorescence from the cuvette without triggering the injector. The outline of reduced transmission very clearly corresponds to the outline of the spray as evidenced by comparison to an averaged LIF image, also given in Fig. 7a. To take this LIF image, the spray was illuminated on the center axis using the counter-propagating laser- beam arrangement. Signals were then recorded with the same camera and filter setup as used for the transmission measurements. Averaged profiles across the LIF and transmission images of the spray in the horizontal direction at $h=25 \mathrm{~mm}$ are shown in Fig. 7b. As is intuitively expected, the results show that the level of extinction varies with the density of the spray. Extinction will be most pronounced within the highdensity regions found in the perimeter of a hollow-cone-type spray, overall leading to the inverse top-hat-like transmission profile as seen in Fig. 7b. The level of attenuation of the signal through the entire spray is found to be about $50 \%$. This means that LIF images, such as in Fig. 7a, lost about $25 \%$ of the original LIF signal to scattering (absorption does not play a role in the present case; see above) since in these measurements the signal light travels only through half of the spray. This estimate assumes a symmetric spray cross-section and attenuation following Beer-Lambert's law.

\section{Spray-structure studies}

\subsection{Horizontal cross-sections}

Images, like that shown in Fig. 4, illustrate that the circular hollow-cone spray cross-section is not as symmetric as expected. To exclude the possibility that the recorded images suffer from attenuation effects even with bi-directional illumination, the injector was rotated by $90^{\circ}$ and $180^{\circ}$, respectively, and averaged LIF and Mie images were taken. After this change, the asymmetric part of the spray shifted by the same angle. Therefore, it is safe to claim that this feature was due to the performance of the particular injector. For further discussion, spatial signal averages over the entire spray cross-section were taken for subsequent analysis. The LIF signals from 3-pentanone can directly be interpreted as proportional to the fuel mass [7]. The evolution of the LIF signal in Fig. 8 thus shows how the amount of fuel mass changes in the cross-section $20 \mathrm{~mm}$ below the injector nozzle as time proceeds. At approximately $0.58 \mathrm{~ms}$ after start of injection control (ASOIC) the spray enters the interrogation region and signals begin to increase rapidly as more fuel enters the cross-section. At $0.68 \mathrm{~ms}$ ASOIC the spray is well developed but then the mass flux decreases rapidly. However, at $1.06 \mathrm{~ms}$ ASOIC, a second increase in mass flux is clearly

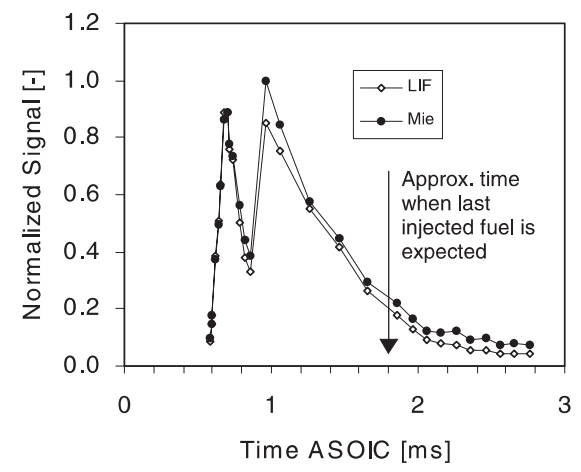

Fig. 8. Evolution of spatially averaged Mie and LIF signals in a horizontal cross-section $20 \mathrm{~mm}$ below the injector nozzle. The expected time for fuel droplets to pass through the laser-sheet plane is approximately given by the first appearance of fuel in the plane and the injection duration of $1.2 \mathrm{~ms}$ 
apparent. It appears that the injection is split into a pre- and main-spray event. This was found to be very repeatable for the injector under study. As the injection duration was set to $1.2 \mathrm{~ms}$, the mass flux through the measurement plane dropped to a low remaining value past approximately $1.8 \mathrm{~ms}$ ASOIC. This can indicate the presence of droplets that remain in this area possibly due to recirculation events. However, even after the times ASOIC $>1.8 \mathrm{~ms}$, for which the injection should be completed, a clear pattern similar to the shape of the fully developed spray cross-section is visible. The arrow in Fig. 8 marks the expected end of fuel flow due to fuel-injection timing. Also shown is the evolution of the spatially averaged Mie-scattering signal. LIF and Mie signals were normalized such that their displayed intensities are equal at the peak of the pre-spray event. While early during the spray evolution the LIF and Mie-scattering signals remain comparable to each other, this changes with increasing time. A closer examination of the ratio of the two signals is therefore discussed below.

As first described by Yeh et al. [4], the ratio of LIF signals and Mie-scattering signals can be related to the Sauter mean diameter of the droplet ensemble under study. The simultaneous LIF and Mie-scattering measurements, performed in this study, can therefore be used to evaluate the dropletdiameter history in the hollow-cone spray as well as means for determining spatial droplet-size distributions. In principle, the scattered-signal intensity $I_{S}$ from a droplet of diameter $D$ can be expressed in terms of the following parameters [15]:

$I_{S}=K D^{n}$ (valid for $D \gg \lambda=$ laser wavelength $)$.

$K$ is a constant that characterizes parameters like signal collection solid angle and detector efficiency etc, while $n$ represents a constant that depends on the nature of the scattering process. Expected diameters for the high-pressure gasoline sprays, such as the one studied here, are on the order of $25 \mu \mathrm{m}$ and therefore with laser light of a wavelength of approximately $0.25 \mu \mathrm{m}$, the condition $D \gg \lambda$ is fulfilled.

The intensity for Mie scattering can be calculated from the Lorentz-Mie theory, and (1) is modified to

$I_{\text {Mie }}=K_{\text {Mie }} D^{2}$.

This was confirmed by experimental checks as discussed and summarized by Jermy and Greenhalgh [6].

Assuming that the laser illuminates the entire volume of the droplets equally during LIF measurements, the LIF signal $[4,5]$ is then described by the following form of (1):

$I_{\mathrm{LIF}}=K_{\mathrm{LIF}} D^{3}$.

The fluorescent tracer used in this study, 3-pentanone, was added in low concentration to the iso-octane fuel to avoid significant attenuation of the laser beam by individual droplets. A measured absorption coefficient of $45 \mathrm{~mm}^{-1}$ for laser light at $266 \mathrm{~nm}$ and room temperature and atmospheric pressure [16] translated into approximately $25 \mathrm{~mm}^{-1}$ for $248 \mathrm{~nm}$ using the relative absorption spectra found in [7]. For the droplet diameters (few $\mu \mathrm{m}$ ) and tracer concentration $(0.5 \%)$ used in this study the attenuation within a single droplet is negligible. Jermy and Greenhalgh [6] discussed work that shows that the use of high-gain laser dyes for spray
LIF investigations can lead to amplified spontaneous emission at very low tracer concentrations, which would increase the exponent $n$ in (1) to values larger than three. The use of 3-pentanone avoids this problem by having a low gain. A volatile fluorescence tracer also has the advantage of producing LIF signals that are proportional to the volume of the droplets up to complete evaporation. However, mismatches in the evaporation characteristics of the base fuel and the tracer can lead to systematic errors. There are also issues with the ambiguity that the liquid and the vapor phases contribute to LIF signals while one ideally would not want signals from the gas phase. This is addressed further below in this paper. On the other hand, non-volatile tracers like laser dyes will produce LIF signals that are independent of the droplet volume, and eventually for very small droplets the dye concentration can reach very high levels and the behavior of the LIF signal on the transition to complete evaporation of the base fuel is not well characterized.

Based on the discussed issues we expect that for our experimental conditions, using 3-pentanone as tracer and modest evaporation, the ratio of the measured fluorescence and Mie signals is a quantity that scales proportionally to the diameter of the droplets. Typically, for sprays as dense as high-pressure gasoline sprays, the volume that is imaged onto one pixel of the ICCD camera contains more than one droplet. Therefore, the intensity value registered by a pixel represents signals from an ensemble of droplets imaged onto the pixel from the interrogation region with coordinates $(x, y)$. This region in fact is three-dimensional, but since the third dimension is not resolved rather than mapped onto the other two dimensions, the $z$ index is omitted. It has been shown by Yeh et al. [4] that the ratio of LIF and Mie signals from ensembles is related to the Sauter mean diameter as

$S M D=K \frac{I_{\mathrm{LIF}}(x, y)}{I_{\mathrm{MIE}}(x, y)}=K^{\prime} \frac{\sum N_{i}(x, y) D_{i}^{3}}{\sum N_{i}(x, y) D_{i}^{2}}$

where $i$ represents the index of droplets from which signals are imaged onto the pixel. In practice, relative signals will be measured and an experimentally determined calibration constant $K$ for one point within the $S M D$ distribution must be available to allow the interpretation of the signal ratio as absolute $S M D$ value. As the calibration has to be performed for only one data point, relative maps of $S M D$ distributions are readily available for evaluation from a simple ratio of LIF and Mie images even without knowledge of absolute diameters. The relative drop-size distribution can be converted into an absolute scale $S M D$ distribution by calibrating the data with a priori data from a single PDA measurement to determine the value of the constant $K$. The spray from the swirl injector that was studied here was extensively characterized by PDA measurements earlier [17]. However, at that time the measurements were performed with a long time window that will make a direct comparison of these results with the imaging results from this study difficult. The measurements of this work represent snapshots with a time resolution of approximately $20 \mathrm{~ns}$, in contrast to the PDA data that were acquired without gating over the entire spray duration. It was therefore decided to present the data of this work on a relative $S M D$ scale. For general orientation, $S M D$ s in the range of $20-30 \mu \mathrm{m}$ were determined with the PDA measurements, thus confirming the validity of the assumption made for (1). 


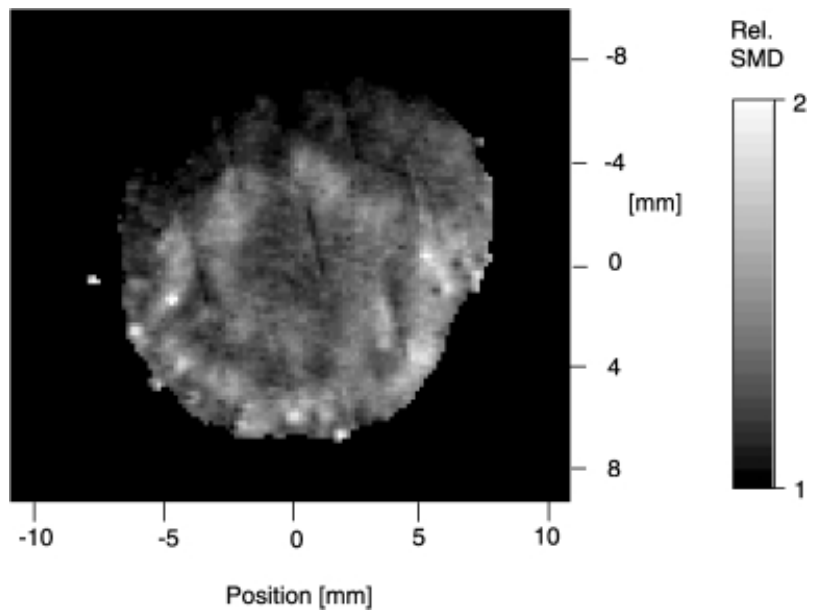

Fig. 9. Instantaneous, relative SMD distribution in a horizontal plane $20 \mathrm{~mm}$ below the injector nozzle, $0.86 \mathrm{~ms}$ ASOIC

The relative $S M D$ distribution that is shown in Fig. 9 was obtained from the ratio of a simultaneously measured pair of single-pulse LIF and Mie images. The spatial variation of the $S M D$ follows the hollow-cone nature of the spray with largest droplet diameters found in the regions of highest mass flux (compare to Fig. 4). Again, an asymmetry of the spray is noticed. Furthermore, one half of the perimeter of the spray frequently exhibits a double-cone structure as in the example of Fig. 9.

The evolution of the spatially averaged $S M D$ at $20 \mathrm{~mm}$ below the injector nozzle is plotted in Fig. 10. A measured spatial variation of $\pm 14 \%$ for the $S M D$ within the horizontal cross-section at a given time leads to the conclusion that the spatially averaged $S M D$ at this location remains constant during the actual injection process, as indicated by the uncertainty limit in Fig. 10. Beyond the actual injection process, i.e. after $1.8 \mathrm{~ms}$ ASOIC, the $S M D$ decreases gradually during this post-spray phase. Note that as the LIF measurement does not distinguish between liquid and gaseous 3-pentanone, the mean droplet diameter can be overestimated, as vaporized tracer will contribute to the measured LIF signal. Therefore, the actual rate of decrease in SMD could be higher. Calculations with KIVA $3 \mathrm{~V}$ [18] show that the expected vapor

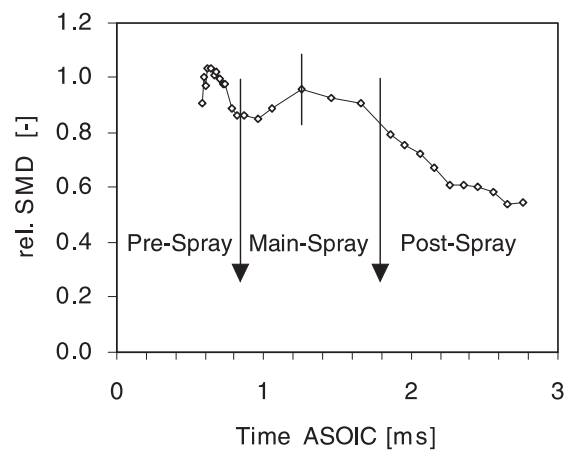

Fig. 10. The spatially averaged relative SMD remains unchanged during the injection period and drops when the main spray event is over. The expected time for fuel droplets to pass through the laser-sheet plane is approximately given by the first appearance of fuel in the plane and the injection duration of $1.2 \mathrm{~ms}$. The error bar indicates the variation of the relative SMD across the horizontal cross-section fraction is on the order of $5-10 \%$ for the sprays and times investigated here, but will significantly increase when studying impinging sprays; see below. This would result in an overestimate of the measured $S M D$ proportional to the measured vapor signal, i.e. up to of $5-10 \%$. Thus, the decrease of the measured droplet diameters is likely to be underestimated.

\subsection{Spray impingement on a flat wall}

Wall impingement of a hollow-cone spray is of particular interest in automotive applications as some direct-injection gasoline-engine concepts make use of an impinging spray on the piston surface to direct the fuel towards the spark plug [19]. To study this process the spray from the Zexel injector was impinged onto a flat metal plate $(38 \times 38 \mathrm{~mm})$ that was placed horizontally $33 \mathrm{~mm}$ below the injector tip. The plate was not heated and thus had the same ambient temperature as the air. Counter-propagating vertical laser sheets illuminated the spray through the center. The interrogation region was a vertical plane, up to 55-mm wide and 15-mm high. As in the case of the transmission measurements, the region of the spray between the injector nozzle and approximately
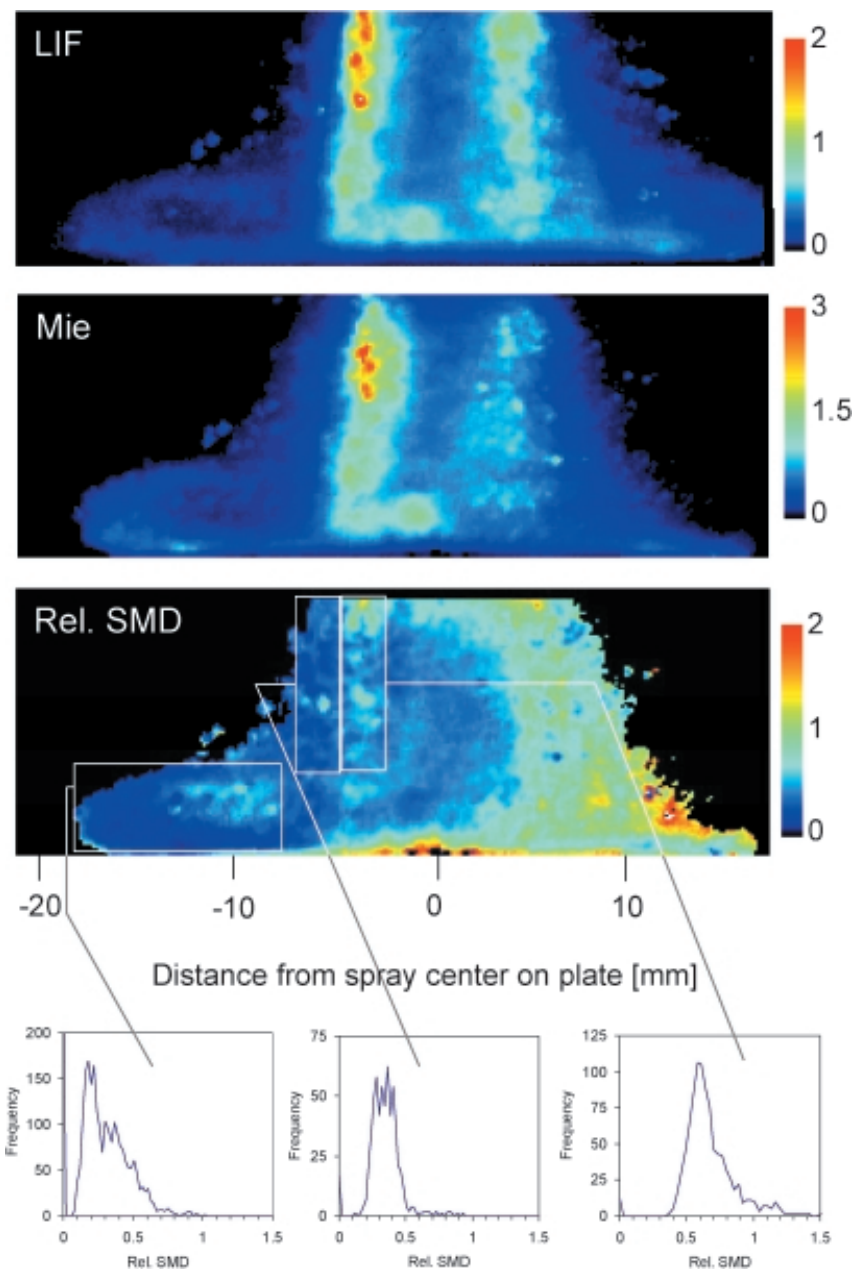

Fig. 11. Single-pulse LIF and Mie images and relative SMD distribution in an impinging spray at $1.46 \mathrm{~ms}$ ASOIC. The plate on which the spray impinges is mounted $33 \mathrm{~mm}$ below the injector nozzle. The gray boxes outline the regions for which the histograms are displayed 
20-mm downstream was blocked from view by the pent roof design of the cylinder head. Droplet-diameter distributions were determined for the impinging sprays in the same manner as discussed above for the horizontal cross-sections. An example of an instantaneous LIF and Mie image pair and the corresponding instantaneous mean droplet diameter distribution is given in Fig. 11 along with three histograms that show the size distribution within the indicated areas. Small droplets with a narrow size distribution characterize the peripheral portion of the spray before impingement. The droplet diameters measured on the 'cone' area of the spray, where the bulk of the mass is transported, have an average value about twice that of the small droplets in the perimeter. Once the spray hits the surface, a broader droplet-size distribution is observed with an average that is substantially lower than the average diameter found in the cone before impingement. This can be attributed to the droplet break up and coalescence after the rebound from the wall. Due to these processes, the droplet-size distribution is wider compared to pre-impingement times. On the right-hand side of the spray a poorer level of atomization is noticed with high relative $S M D$ values, up to twice compared to values found in the left-hand side of the spray. This underlines again that the spray is not symmetric, as already discovered in the measurements of horizontal cross-sections, shown in Fig. 4. It should be noted that vapor levels of up to $25 \%$ in this region are expected from a computational study of the spray [18]. This will result in $S M D$ values that are correspondingly overestimated, as the LIF signals will include contributions from the vapor phase.

Averaged $(N=25)$ LIF and Mie images were analyzed as well and $S M D$ distributions were determined for the same conditions as shown in Fig. 11. Differences are noted in the smoothness of the distributions but also in the structure of the right-hand side of the spray (see Fig. 12). It appears from the images that the 'cone' part of the spray is much broader on
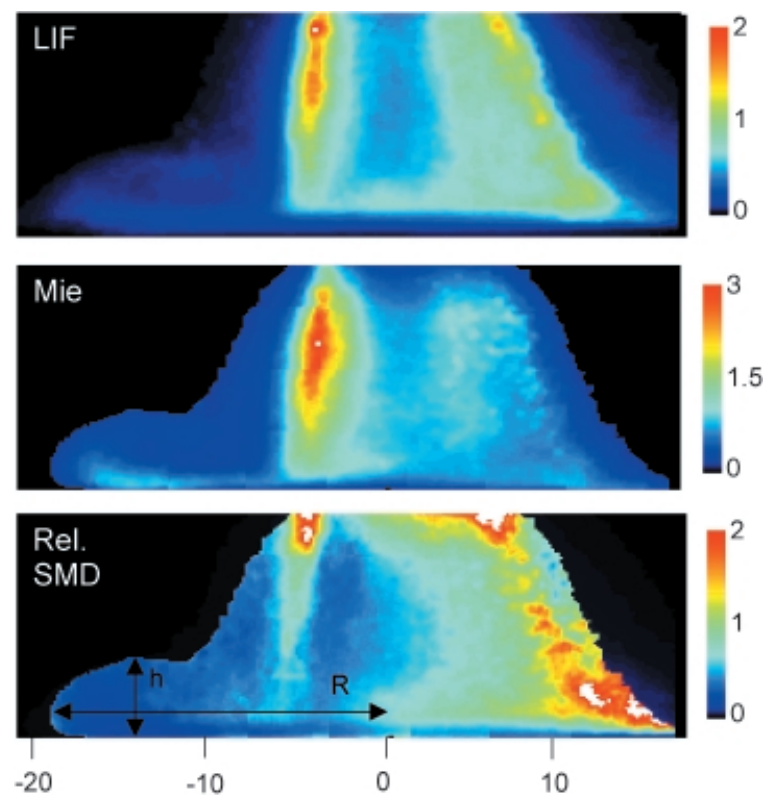

Distance from spray center on plate [mm]

Fig. 12. Averaged $(N=25) \mathrm{LIF}$ and Mie images were used to compute the relative SMD distribution of an impinging spray at $1.46 \mathrm{~ms}$ ASOIC this side of the spray. This is the result of occasional doublecone injections as discussed in the context of the horizontal cross-sections. Histograms of the SMD taken in the same regions as shown in Fig. 11 for an individual injection have the same general structure.

The radial extent $R$ of the roll-up vortex, formed after the wall impingement, is measured from the spray axis to the outermost position of the roll-up vortex, while the height $h$ of the vortex is the upward distance from the wall to the highest point of the vortex (see Fig. 12 for schematic). The motion of the vortex on the left-hand side of the spray is plotted in Fig. 13. Velocities of $22 \mathrm{~ms}^{-1}$ for the spray-tip motion in the radial direction and $6 \mathrm{~ms}^{-1}$ for the vertical motion of the vortex were determined for the spray conditions as given above. This compares to approximately $30 \mathrm{~ms}^{-1}$ for the spray before it hits the surface. The variance in the radial spray-tip position $R$ - as measured from the center to the left-hand side - is generally small as indicated by the error bars in Fig. 13, implying that although the performance of the injector in terms of radial symmetry is poor, at least it is consistent on the left-hand side. In contrast to this, the variation of the vortex height $h$ increases substantially for later times.

Approximately $1 \mathrm{~ms}$ ASOIC the spray impinges on the plate surface to form a liquid-fuel film. Thereafter, fuel droplets and a liquid film spread in a radial direction along the wall, coupled with a formation of a roll-up vortex. During studies of repeated impingements on an initially dry surface,

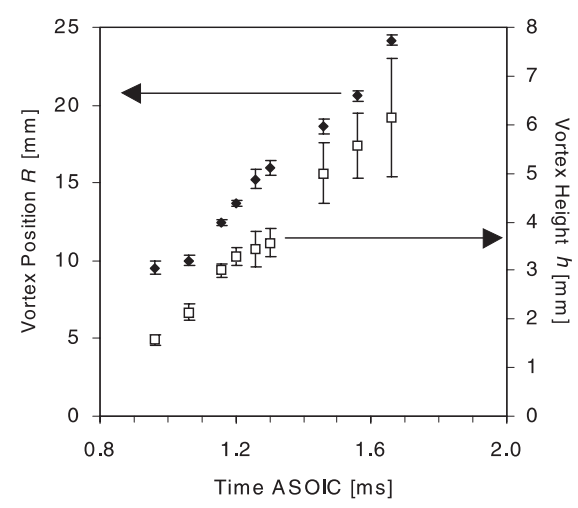

Fig. 13. Radial and vertical extent of the roll-up vortex on the left-hand side of the impinging spray. Error bars represent the standard deviation of the measurement

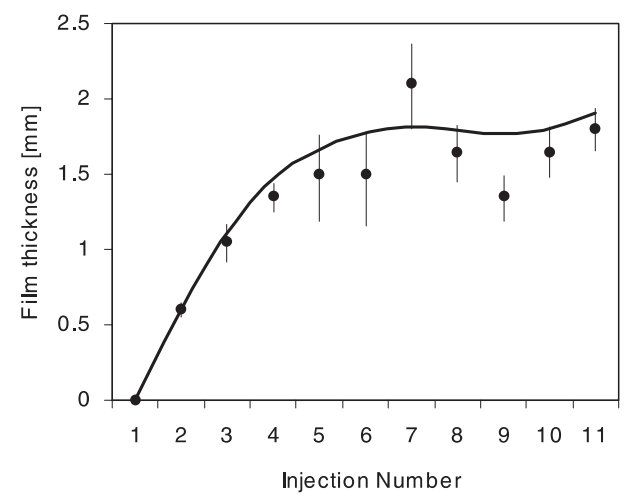

Fig. 14. Film-thickness measurements (one injection per second at ambient pressure and room temperature). Error bars are given as standard deviations of the measurements 
the build-up of the fuel film could be characterized. LIF signals close to the surface were measured with a side view of the plate. The thickness of the film could be directly read from vertical profiles through the LIF images. The thickness increased with increasing number of impingements reaching asymptotic values after about 10 impingements. This indicates the buildup of a liquid film that reaches a steady-state thickness of $1.7 \mathrm{~mm}$ after a few injections (see Fig. 14). Since the measurements were conducted at room temperature, the evaporation rate is low and the film can reach a substantially larger thickness as would be expected in an engine.

\section{Conclusions}

The design and implementation of a robust acquisition system that allows measurements of spectrally isolated planar Mie-scattering and LIF signals on a single ICCD camera was described. This setup, together with bi-directional lasersheet illumination, was used to investigate the structure of an automotive hollow-cone-type spray. Although a reduction of spatial resolution results from the combined imaging of both signals on one camera chip, a sufficient level of detail of the investigated physical characteristics of a hollow-cone spray was attained. The measurements were carried out under ambient conditions, i.e. room temperature and atmospheric pressure. However, the injector was mounted in a real engine cylinder head to mimic optical properties of an optical direct-injection engine. The fuel used was iso-octane with $0.5 \%$ 3-pentanone added as fluorescence tracer. Sources of signal interference were investigated first. Significant reflections of signal light, especially for LIF signals, were noticed for a horizontal light-sheet arrangement. Vapor blasting of the cylinder-head surface considerably reduced the reflections of LIF signals from the aluminum cylinder head surface. Reflections of Mie signals are less problematic since the uncoated aluminum surface is significantly less reflective at $248 \mathrm{~nm}$ compared to $400 \mathrm{~nm}$ where the LIF signals occur. A counterpropagating laser-beam arrangement reduces the influence of laser-light extinction, induced by the dense nature of the spray. A method for characterizing the level of signal extinction between the spray and the camera was described and used to find that signals are attenuated by as much as $25 \%$ in the spray that was investigated here.

The time-dependent mass distribution of fuel in a horizontal cross-section $20 \mathrm{~mm}$ below the injector nozzle showed the existence of a pre- and main spray. From the ratio of LIF and Mie-scattering signals information about the evolution of the Sauter mean diameter was derived. This showed the presence of either recirculated droplets or droplets that arrive late and evaporate during the time of the measurement. Images of an impinging spray were analyzed to yield information about the propagation velocity of the roll-up vortex and the time-dependence of the thickness of the liquidfuel film that builds up upon impingement. The influence of impingement on the $S M D$ was characterized for instantaneous and averaged measurements. In the single-pulse, as well as averaged, $S M D$ distributions the droplet break up after impingement of the spray was observed as evident from histograms of the $S M D$ in respective regions.

Acknowledgements. This research was sponsored by US Department of Energy contract nos. DE-FG04-99AL66236, DE-FG02-98ER14915 - subcontract no. 14412-S1, Division of Chemical Sciences, Office of Basic Energy Sciences and the General Motors Corporation through the General Motors Satellite Research Laboratory at the University of Michigan. The authors also acknowledge the contributions of Dr. M.C. Drake and Dr. D.L. Harrington.

\section{References}

1. M. Rachner, M. Brandt, H. Eickhoff, C. Hassa, A. Bräumer, H. Krämer, M. Ridder, V. Sick: Proc. Combust. Instrum. 26, 2741 (1996)

2. W.D. Bachalo, M.J. Houser: Opt. Eng. 23, 583 (1984)

3. G. Wigley: Optical Diagnostics for Flow Processes (Plenum, New York 1994)

4. C.-N. Yeh, H. Kosaka, T. Kamimoto: Prog. Astronaut. Aeronaut. 12, 297 (1996)

5. J. Zelina, A. Rodrigue, S.V. Sankar: AIAA Meeting, Reno, NV (1998) paper $98-0148$

6. M.C. Jermy, D.A. Greenhalgh: Appl. Phys. B 71, 703 (2000)

7. F. Grossmann, P.B. Monkhouse, M. Ridder, V. Sick, J. Wolfrum: Appl. Phys. B 62, 249 (1996)

8. F. Ossler, M. Alden: Appl. Phys. B 64, 493 (1997)

9. C. Preussner, C. Doering, S. Fehler, S. Kampmann: SAE Tech. Pap. Ser. 980498 (1998)

10. A.J. Smith (Ed.): The Book of Photon Tools (Oriel Instruments, Stratford, CT, USA 2000)

11. S. Einecke, C. Schulz, V. Sick: Appl. Phys. B 71, 717 (2000)

12. V. Sick, B. Stojkovic: Appl. Opt. 40(15), 1 (2001)

13. J. Su, M.C. Drake, T.D. Fansler, D.L. Harrington: 11th Annu. Conf. Liquid Atomization and Spray Systems, Institute for Liquid Atomization and Spray Systems, ilass Americas '98, Sacramento, CA, 17-20 May, 1998

14. D.G. Talley, J.F. Verdieck, S.W. Lee, V.G. McDonnell, G.S. Samuelsen: AIAA Meeting, Reno, NV (1996) paper 96-0469

15. G.F. Bohren, D.R. Huffman: Absorption and Scattering of Light by Small Particles (Wiley, New York 1983)

16. M.-T. Lin: University of Michigan, private communication (2000)

17. D.L. Harrington: General Motors Corporation, private communication (2001)

18. B. Vanzieleghem: University of Michigan, private communication (June 2000)

19. F.-Q. Zhao, M.-C. Lai, D.L. Harrington: Prog. Energy Combust. Sci. 25, 437 (1999) 